\title{
Subtalar Arthroereisis with Endorthesis in Adult-acquired Flatfoot: Classification of the Postoperative Rehabilitation Phases
}

\author{
by Massimiliano Polastri, MSc, PT ${ }^{1}$, Alessandro Graziani, MSc, PT $^{1}$, Stefano Cantagalli, MD $^{2}$
}

The Foot and Ankle Online Journal 5 (4): 1

Flatfoot is a biomechanical condition in which the medial longitudinal arch collapses, causing flattening of the foot towards the ground. In adult-acquired flatfoot, the subtalar joint has a greater range of motion than a normal foot, and multiple factors can cause the onset of this condition. Subtalar arthroereisis with endorthesis is a surgical procedure by which an implant is positioned in the sinus tarsi depression in order to limit the excessive pronation of the subtalar joint. Subtalar arthroereisis is often associated with adjunctive procedures. A period of three weeks of non-weight bearing is recommended after surgery and additional protection is achieved as the load is increased. In order to be able to discuss the postoperative course, it is useful to be able to classify it. Basically, the classification proposed in this paper is a practical/theoretical instrument which seeks to contribute to a better understanding and achievement of the aims and outcome desired at each stage described. Postoperative rehabilitation must be oriented to both protect the surgical site and to enhance foot mobility. We have proposed a classification of the rehabilitative pathway after subtalar arthroereisis with endorthesis based on our experience, also considering the related literature. Furthermore, we provide a synthetic description of the surgery, and the rehabilitation techniques are discussed. The ultimate goal of the rehabilitation project is centered on obtaining the physical condition closest to that required for the daily activity of the healthy population with the aim of returning to full recovery after surgery. To this end, a certain degree of multiprofessional cooperation is always recommended in order to ensure patient safety and obtain the best results.

Key words: Flatfoot, Gait, Prostheses and Implants, Rehabilitation, Subtalar Joint, Surgical Cast, WeightBearing.

Accepted: March, $2012 \quad$ Published: April, 2012

This is an Open Access article distributed under the terms of the Creative Commons Attribution License. It permits unrestricted use, distribution, and
reproduction in any medium, provided the original work is properly cited. OThe Foot and Ankle Online Journal (www.faoj.org), 2011 All rights reserved.

Address correspondence to: Massimiliano Polastri, Physical Medicine and Rehabilitation, Bologna University Hospital, Sant' Orsola-Malpighi Polyclinic, Via G. Massarenti, 9. 40138 - Bologna, Italy.

Email: gbptap1@gmail.com

\footnotetext{
${ }^{1}$ Physical Medicine and Rehabilitation, Bologna University Hospital, Sant' Orsola-Malpighi Polyclinic, Bologna, Italy.

${ }^{2}$ Orthopedics and Traumatology, Bologna University Hospital, Sant' OrsolaMalpighi Polyclinic, Bologna, Italy.
}

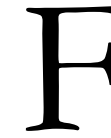
latfoot $(\mathrm{FF})$ is a biomechanical condition in which the medial longitudinal arch collapses causing flattening of the foot towards the ground. The onset of this disorder may occur at different ages from the first years of life up to adulthood. 
In adult-acquired flatfoot (AAF), the subtalar joint (STJ) has a greater range of motion than a normal foot, and multiple factors can cause the onset of this condition. ${ }^{1}$ The STJ is a diarthrosis (trochoid) which connects, in two different locations (separated by the sinus tarsi depression), the posterior-inferior surface of the talus with the superior face of calcaneus and the anterior-inferior part of the talus with the anterior-superior surface of the calcaneus. The articular activities possible at this level are adduction and abduction of the rearfoot, associated with the supination and pronation of the foot, respectively. The realization of these complex movements is facilitated by Chopart's joint (midtarsal). ${ }^{2}$ Arthroereisis is a surgical procedure which limits the amount of motion possible in a joint which has become excessively mobile. ${ }^{3}$ In subtalar arthroereisis (SA) with endorthesis, an implant is positioned in the sinus tarsi depression in order to limit the excessive pronation of the STJ, which is typically present in AAF. ${ }^{4-6}$ Although it has proven to be an effective surgical technique, certain complications are described in the literature..$^{7-10}$ Essentially, in the past, major interest was focused on pediatric patients. ${ }^{11,12}$ More recently, Evans (2008) has defined a therapeutic algorithm for rehabilitation treatment in children. ${ }^{13}$ Originally, the insert of an expandable orthosis was used for the treatment of pediatric flat foot. ${ }^{14-16}$ Characteristics of the implants are discussed in the literature. ${ }^{17}$ Evans and Rome (2011), have found that there is limited evidence of the efficacy of nonsurgical intervention in children with flexible flat feet. In their research, these authors have also provided a wide and complete overview of the surgical approaches available, including arthroereisis. ${ }^{18}$ As introduced above, pronation is one of the movements (together with supination) possible at the subtalar level; one must be aware of this because valgus of the rearfoot characterizes AAF. Postan et al. (2011) have discussed the association of the anatomical variations of the spring ligament and sustentaculum tali with the risk of developing AAF. ${ }^{19}$ Chang and Lee (2007) have provided a careful description of the kinematics, the surgical treatment, and the indications and contraindications as well as the postoperative management, and have described one condition which causes flexible AAF namely: the posterior tibial tendon dysfunction.
For the correction of $\mathrm{AAF}$, adjunctive procedures are often carried out using SA. A period of three to four weeks of non-weight bearing in a cast is recommended after surgery and additional protection is achieved with the use of a walker for more three weeks as the load is increased. ${ }^{20}$ The main purpose of this study was to classify the rehabilitation phases after surgical correction of AAF by means of SA with endorthesis. The literature was reviewed to identify studies which have investigated postoperative rehabilitation after SA. To our knowledge, no previous papers have been published on this matter.

\section{Surgery at a glance}

We herein describe the procedure of subtalar arthroereisis with endorthesis in association with additional procedures on soft tissues for the treatment of painful and flexible AAF. ${ }^{5,20}$ Subtalar arthroereisis locks the sliding between the talus and the calcaneus, restoring their positions; an implant (ProStop ${ }^{\circledR}$, Arthrex Inc., Naples, FL 34108, US) is inserted within the sinus tarsi determining the reduction of the pronation of the STJ acting as a self-locking wedge, according to Vogler's classification. ${ }^{21}$ This system is composed of titanium cannulated screws, threaded and conical in shape, of different sizes ( 7 to $12 \mathrm{~mm}$ ) and lengths (12 to $16 \mathrm{~mm}$ ) so that they can be precisely adapted to the tarsal canal. The anesthesia is generally spinal and specific for the limb operated on. It is carried out by injecting anesthetic into the subarachnoid space with a 25 Gauge needle by means of a injection of the dura mater and of the arachnoid in the lumbar spaces below L2. To this end, the patient is positioned in a sitting position or in lateral decubitus, and the procedure is performed aseptically. Before placing the patient on the operating table, one must wait approximately 5-10 minutes to evaluate the level of the anesthesia. The surgical technique is performed with the patient in a supine position on the operating table with a tourniquet at the root of the thigh root after inserting the limb into an Esmarch bandage. The tourniquet is applied to induce lower limb ischemia so as to create a bloodless field in order to better identify the vascular structures, nerves and tendons. 
An incision of approximately two $\mathrm{cm}$ on the lateral portion of the sinus tarsi is made, allowing the insertion of a guide wire between the two beams of the talar-calcaneal ligament and the interosseus ligament; this facilitates the introduction of a size tester. Under fluoroscopy, the correct implant dimension is determined and the surgeon can proceed with the insertion of the screw using a screwdriver until, the screw itself, is level with the outer edge of side wall of the talus neck. The guide wire is removed and a stitch is applied. At this point, the tension of the triceps tendon is evaluated and, if necessary, a Hoke's percutaneous tenotomy is performed to achieve the appropriate dorsal flexion of the ankle joint. $^{22}$ Subsequently, to correct the talus protrusion, an additional internal procedure of tensioning of the posterior tibial tendon is carried out: $:^{23,24}$ an incision of approximately four $\mathrm{cm}$ is made on the navicular tuberosity, the tendon is detached from the navicular tubercle maintaining the plantar extension of the fibers, and the periosteum is dissected. The prominence of the navicular is then tangentially excised and, if present, accessory bone is removed; tenolysis, repair and tensioning of the posterior tibial tendon are performed at this point. The surgery, including the additional procedures as described above, requires approximately 60 minutes.

\section{Rehabilitation phases}

Antithrombotic prophylaxis is managed at home with low molecular weight heparin for thirty days after surgery. During the postoperative course, the patient must use a walker for thirty-five days and walk without weight bearing for the first three weeks. Between days twenty-one and thirty-five, the load is progressively increased. In order to be able to discuss the postoperative course after SA with endorthesis, it is useful to be able to classify it. Basically, the classification proposed in this study is a practical/theoretical instrument which may help professionals to better understand and achieve the aims and outcome desired at each stage described. Each phase must be carefully evaluated both physically and clinically.

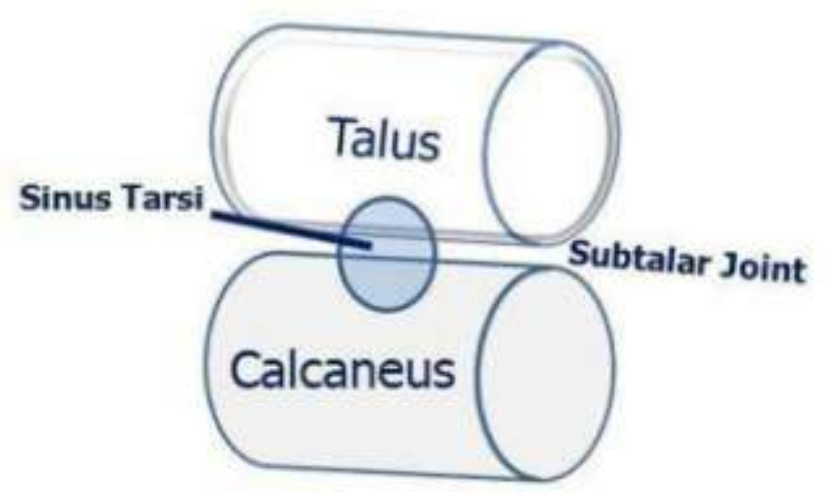

Figure 1 Schematic representation: sinus tarsi is an anatomical space present between the talus (top) and the calcaneus (bottom). Due to the anatomical surfaces, the two cylinders can roll one upon the other (pronation and supination).

If we think of the STJ as two overlapped cylinders, we immediately realize that, at this level, the maximum range of motion is possible in the transverse plane: the cylinders can thus produce movements of pronation and supination. Conversely, flexion and extension are limited due to the anatomical surfaces (Fig. 1).

\section{Stage 1 (immobilization and pain)}

After surgery, patients are advised not to put weight on the foot and to use a walker in order to protect the surgical site for a period of three weeks. In this initial phase, the patient is likely to be overcautious and have some degree of fear, in carrying out the usual daily activities. Both, pain and infection prevention procedures are similar to those provided in arthroscopic surgery of the ankle. ${ }^{25}$ Conversely, patients undergoing ankle arthroscopic excision do not wear a cast after surgery and they are advised to limit the range of motion and to protected the load in the first twenty-four hours postoperatively. ${ }^{26}$ On the other hand, in surgical procedures of the ankle more invasive than SA, patients are encouraged to exert weight after surgery. ${ }^{27}$ 


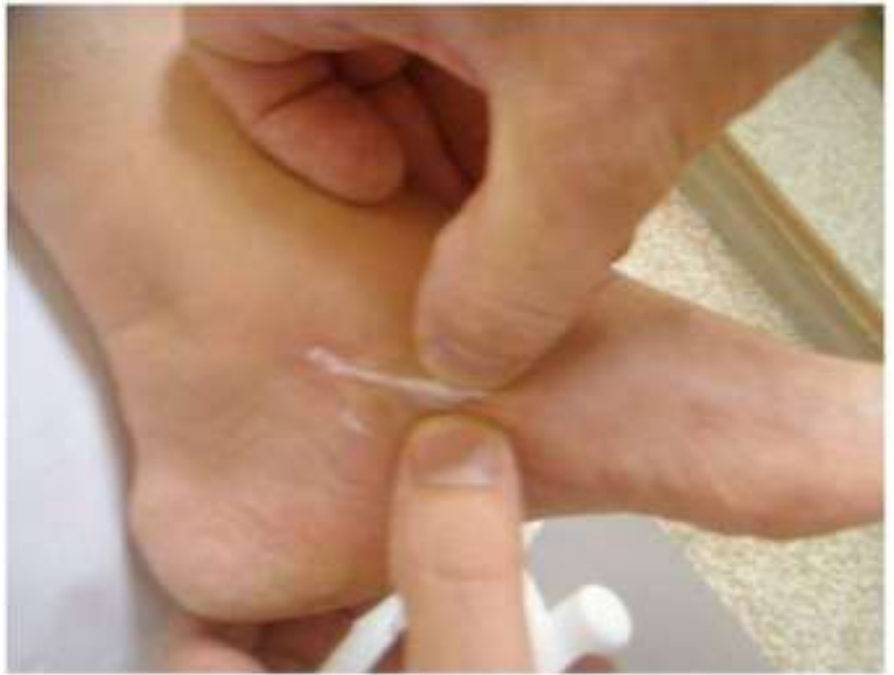

Figure 2 Scar massage on the surgical access of the posterior tibial tendon.

In patients undergoing $\mathrm{SA}$, if a physiotherapist is involved in stage 1 , in order to prevent/manage any complications, he/she must be present as a counselor and must refer every unusual condition (after clinical evaluation) which may compromise the postoperative course. When no red flags (severe pain, heat, inability to walk even with the walker) are present, the rehabilitation activity is limited to observation and advice (sleeping with minimum elevation of the feet putting a pillow under the mattress, walking short differences but often, maintaining proper alignment of the pelvis through the correct use of crutches, noting if there is blood in the dressing, regularly using the walker). Orthopedic procedures are recognized as the most painful because of the need to walk. ${ }^{28}$ In stage 1, pain should be low to moderate and no additional treatment is usually required. Otherwise, the patient must be referred to a physician.

\section{Stage 2 (edema, pain and weight-bearing)}

When the dressing is removed after 35 days, the physiotherapist must check for the presence of edema. If, in the previous stage, pain control was obtained by means of anti-inflammatory drugs or painkillers, the residual -algic conditions must now be evaluated and eventually treated by referring the patient to a physician.

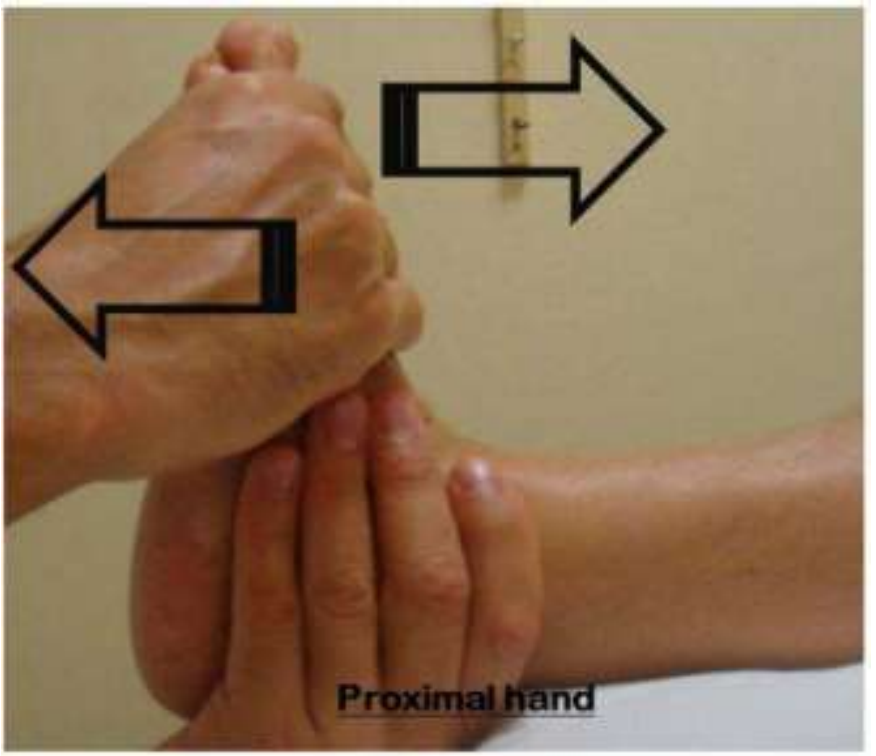

Figure 3 The physiotherapist's proximal hand blocks the STJ to limit eversion and inversion of the foot whereas plantar and dorsal flexion are performed.

Permanence of the -algic symptoms represents a complication assuming that, after one month, in almost all cases, pain should be at a minimum. Nevertheless, a certain degree of discomfort is present during or after walking/standing. Furthermore, in this period, which covers the first 3550 days, the presence or absence of possible algodystrophy must be evaluated. Tenderness, vascular instability, stiffness and swelling, if present, are red flags for this issue. On the other hand, if the foot is not swollen, not hot and not painful, the edema (if present) can be treated through massage, and elevation at night. Thanks to the surgical technique, manual treatment of the sinus tarsi scar is not usually necessary (minimal access); conversely, manual massage is performed to facilitate the disappearance of the scar on soft tissue procedure sites using an elasticizing cream $\left(\right.$ Rilastil $^{\circledR}$ Laboratori Milano, Istituto Ganassini S.p.A. di Ricerche Biochimiche, 20139 Milano, IT) (Fig. 2). Usually, at the end of stage 2, the patients no longer need a walker and/or crutches. Weight bearing is progressively allowed and the patient can wear sport shoes. Articular mobilization should be pursued at the following levels: talocrural joint and forefoot. 


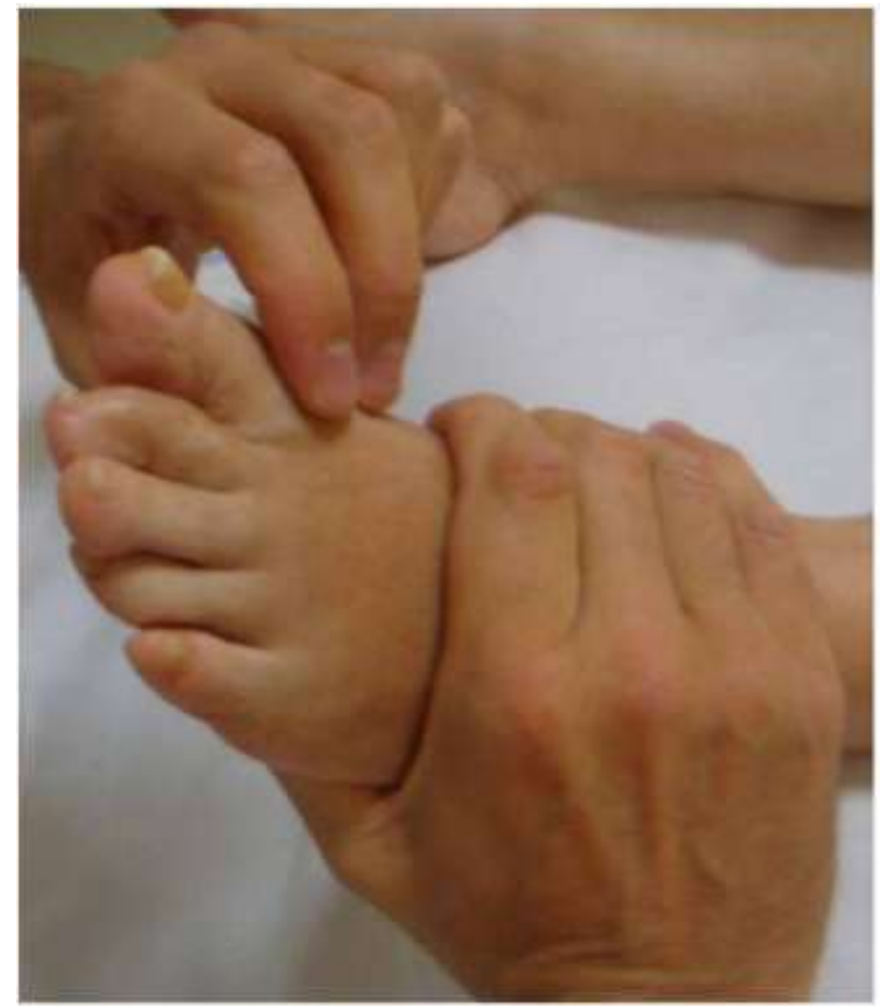

Figure 4 Passive movement of the first ray with the STJ blocked by the proximal hand.

To protect the implanted endorthesis, pronation and supination of the STJ are not required or even encouraged. As described above, this diarthrosis would be comparable to a pair of overlapped cylinders which move around the longitudinal axis of the foot. Why force pronation or supination at this level when, after surgery, they are protected? Conversely, mobilization of the areas peripheral to the surgery are recommended in order to achieve the maximum interest on the part of the patient in recovery. Plantar and dorsal flexion of the ankle and mobilization of the forefoot are carried out with the patient in a supine position with the knee supported in flexion: block the STJ with the proximal hand to avoid pronation or supination at this level during articular recruitment (Figs. 3 and 4). The proprioceptive component is important at this stage and should be composed of several levels, compatible with weight bearing. The manual approach should start with closed kinetic chain exercises stimulating coordinated muscle contraction in the articular segments of the lower limb increasing the capsularligament stability of the ankle itself.

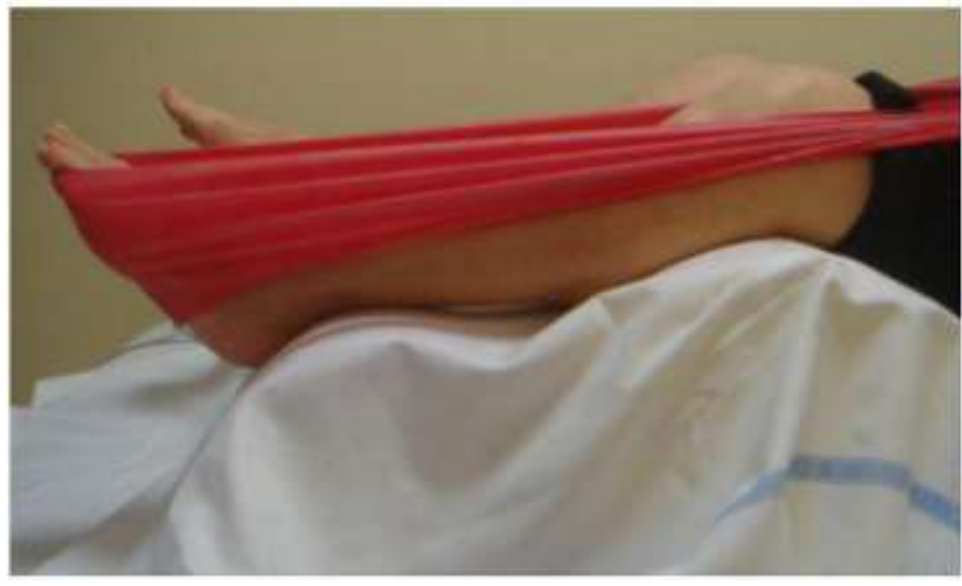

Figure 5 Muscular self-administered strengthening in a supine position (plantar-dorsal flexion) using a TheraBand $^{\circledR}$.

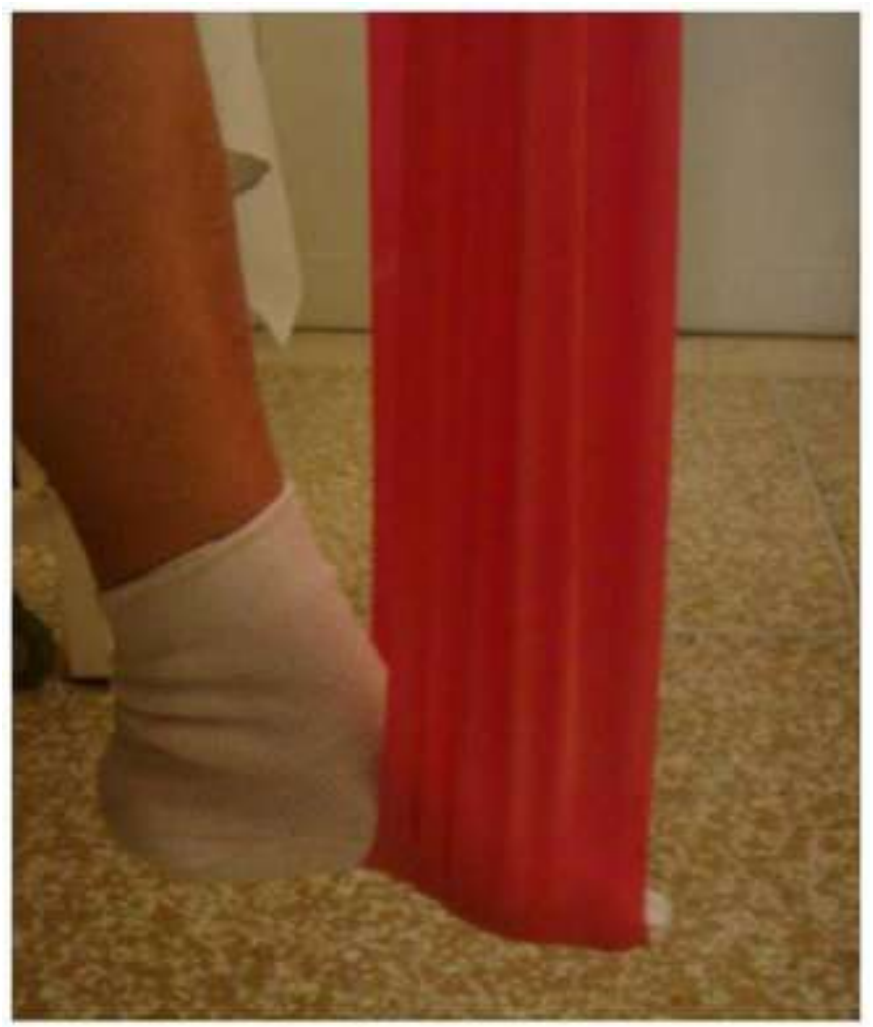

Figure 6 In a sitting position, the patient is encouraged to carry out movements in all directions regulating/increasing the elastic resistance with their own hands. 
In addition to developing muscle strength, these exercises optimize the functional capacity of the individual by encouraging recovery of the physiological activities of the joint operated on. Closed kinetic chain exercises take advantage of the normal joint structure, and the entire proprioceptive system is stimulated. To perform exercises with patient in a supine position, the ankle is placed in a side panel in front of the subject. Making the first movements in an anterior-posterior direction is encouraged to recreate and improve neuromuscular coordination; the same movements are necessary in the lateral direction and then combined across multiple directions, always with the foot in contact with the wall. Once the patient is capable of tolerating an increased load, the physiotherapist can propose the same procedure with the patient first sitting and then standing with the foot resting on the ground. As this stage is focused on proprioception, unstable balance tools should be used in order to enhance both dorsal and plantar flexion.

\section{Stage 3 (mobility and gait)}

At approximately 50 days, assuming that pain and edema have been resolved or are in resolution, the patient must be encouraged to increase mobility. Exercises and mobility techniques are continue using an elastic band (Thera-Band $^{\circledR}$, The Hygenic Corporation, Akron, $\mathrm{OH}$ 44310, US) to develop progressive resistance in the various planes of motion (Figs. 5 and 6). The patient should be instructed and encouraged to do these exercises even in the absence of the physiotherapist, the so-called phase of self treatment at home is essential at this point in order to optimize the timing and results. ${ }^{29}$ Hupperets, et al., (2009) have found that unsupervised proprioceptive home based training could benefit the general population. ${ }^{30}$ Basically, rehabilitation at this stage is still focused on proprioception using unstable balance tools with both unilateral and bilateral weight bearing. The final phase of muscular strengthening is dedicated to all antigravity movements in which the foot is in a challenging biomechanical context. The patient undergoes a series of exercises which are in contrast to the body weight acting together with gravity.
The starting point of these exercises begins with the full load step on the limb which was operated on; key element for ensuring recovery of the physiological gait. More challenging related exercises are represented by walking uphill with a gradual slope (for example treadmill), climbing the stairs one or two steps at a time and then coming down the stairs (to stimulate the eccentric component of the muscle contraction). Both strength and antigravity exercises are recommended to achieve the full recovery and return to the normal activity. In this phase, the patients should be referred to hydrokinesitherapy to maximize results and improve the postoperative outcome. Berger, et al., (2006), comparing the immediate effects of standard physiotherapy and balneotherapy on postural capacity in subjects with lower limb injuries, observed that exercising under water could reinforce proprioceptive input. ${ }^{31} \mathrm{~A}$ good recovery of foot function can be achieved by proposing implementation of walking synergies such as walking backwards on one's own toes, walking on heels or cross stepping.

The help of a mirror can provide valuable visual feedback in order to correct any altered patterns. One must research transition from normal to faster walking and then to running. The ultimate goal of the rehabilitation project is centered on obtaining the best physical condition closest to the daily activity of the healthy population with the aim of returning to full recovery after surgery.

\section{Stage 4 (return to sports activities)}

The last phase of antigravity training is completed with proper exercises such as jumping in place or on a trampoline. A gradual resumption of the sports activity previously carried out is allowed and a radiographic check-up is required to verify the implant positioning. 


\section{Conclusions}

Despite the fact that SA was initially proposed for pediatric patients, it is being an increasingly used procedure in the adult population. In this paper we have proposed a classification of the rehabilitative pathway after SA based on our experience also considering the related literature. The main limit of our classification is represented by the absence of a sample with which to make statistical comparisons. Nevertheless, we wanted to address the matter when we realized the need for clarifying and classifying a patient's physical condition after corrective surgery. Again, even with its limits, this overview should help and stimulate further research. After subtalar arthroereisis with endorthesis, postoperative rehabilitation must be oriented both to protect the surgical site and to enhance mobility of the foot. In order to maximize results and contain clinical risk, the physiotherapist must be able to carry out a functional evaluation and, if necessary, refer patients when complications occur. To this end, a certain degree of multiprofessional cooperation is always recommended in order to ensure patient safety and achieve the best results.

\section{References}

1. Needleman RL. Current topic review: subtalar arthroereisis for the correction of flexible flatfoot. Foot Ankle Int 2005 26: 336-346. [PubMed]

2. Ceccaldi A. Pratique de la rééducation du pied. Paris: Masson, 1967. Website]

3. Churchill's Medical Dictionary. New York: Churchill Livingstone, 1989 , p. 163.

4. Highlander P, Sung W, Weil L. Subtalar arthroereisis. Clin Podiatr Med Surg 2011 28: 745-754. [PubMed]

5. Arangio GA, Reinert KL, Salathe EP. A biomechanical model of the effect of subtalar arthroereisis on the adult flexible flat foot. Clin Biomech 2004 19: 847-852. PubMed]

6. Saxena A, Nguyen A. Preliminary radiographic findings and sizing implications on patients undergoing bioabsorbable subtalar arthroereisis. J Foot Ankle Surg 2007 46: 175-180. PubMed

7. Van Ooij B, Vos CJ, Saouti R. Arthroereisis of the subtalar joint: an uncommon complication and literature review. J Foot Ankle Surg 2012 51: 114-117. [PubMed]
8. Corpuz M, Shofler D, Labovitz J, Hodor L, Yu K. Fracture of the talus as a complication of subtalar arthroereisis. J Foot Ankle Surg 2012 51: 91-94. PubMed]

9. Rockett AK, Mangum G, Mendicino SS. Bilateral intraosseous cystic formation in the talus: a complication of subtalar arthroeresis. J Foot Ankle Surg 1998 37: 421-425. PubMed]

10. Oloff LM, Naylor BL, Jacobs AM. Complications of subtalar arthroereisis. J Foot Surg 1987 26: 136-140. [PubMed]

11. De Doncker E. Treatment of static flatfoot. I. Orthopedic treatment: kinesitherapy. Rev Chir Orthop Reparatrice Appar Mot 1977 63: 756-757. PubMed]

12. Fregnani L, Droghetti I. Corrective gymnastics in the treatment of flatfoot in children. Arcisp S Anna Ferrara 1969 22: 629-640. [PubMed]

13. Evans AM. The flat-footed child - to treat or not to treat: what is the clinician to do? JAPMA 2008 98: 386-393. PubMed] 14. Giannini S, Girolami M, Ceccarelli F. The surgical treatment of infantile flat foot. A new expanding endo-orthotic implant. Ital J Orthop Traumatol 1985 11: 315-322. [PubMed]

15. Gutiérrez PR, Lara MH. Giannini prosthesis for flatfoot. Foot Ankle Int 2005 26: 918-926. [PubMed]

16. Giannini S, Ceccarelli F, Benedetti MG, Catani F, Faldini C. Surgical treatment of flexible flatfoot in children a four-year follow-up study. JBJS 2001 83A(Suppl 2): 73-79. [PubMed]

17. Villani C, Chiozzi F, Persiani P, Costantini A. Flat foot: a comparison of surgical methods. Chir Organi Mov 2003 88: 4955. [PubMed]

18. Evans AM, Rome K. A Cochrane review of the evidence for non-surgical interventions for flexible pediatric flat feet. Eur J Phys Rehabil Med 2011 47: 69-89. [PubMed]

19. Postan D, Carabelli GS, Poitevin LA. Spring ligament and sustentaculum tali anatomical variations: anatomical research oriented to acquired flat foot study. FAOJ 2011 4: 1. Website]

20. Chang TJ, Lee J. Subtalar joint arthroereisis in adult-acquired flatfoot and posterior tendon dysfunction. Clin Podiatr Med Surg 2007 24: 687-697. [PubMed]

21. Maxwell JR, Cerniglia MW. Subtalar joint arthroereisis. In: Bank AS, Downey MS, Martin DE, Miller SJ. McGlamry's Comprehensive Textbook of Foot and Ankle Surgery. $3^{\text {rd }}$ ed. Philadelphia: Lippincott Williams \& Wilkins, 2001, vol. 1, pp. 901-914.

22. Hoke M. An operation for stabilizing paralytic feet. J Orthop Surg 1921 3: 494-507. [Website]

23. Gould JS. Direct repair of the posterior tibial tendon. Foot Ankle Clin 1997 2: 275-279.

24. Giannini S, Vannini F, Bevoni R, Romagnoli M, Di Gennaro V. Trattamento chirurgico delle lesioni del tibiale posteriore. In: Progressi in medicina e chirurgia del piede. Bologna: Timeo, 2007 16: 73-87.

25. Bonasia DE, Phisitkul P, Saltzman CL, Barg A, Amendola A. Arthroscopic resection of talocalcaneal coalitions. Arthroscopy 2011 27: 430-435. [PubMed] 
26. Noguchi H, Ishii Y, Takeda M, Hasegawa A, Monden S, Takagishi K. Arthroscopic excision of posterior ankle bony impingement for early return to the field: short-term results. Foot Ankle Int 2010 31: 398-403. PubMed]

27. Talarico LM, Vito GR, Zyryanov SY. Management of displaced intraarticular calcaneal fractures by using external ring fixation, minimally invasive open reduction, and early weight bearing. J Foot Ankle Surg 2004 43: 43-50. [PubMed]

28. D'Amours RH, Ferrante FM. Postoperative pain management. JOSPT 1996 24: 227-236. [PubMed]

29. Mikesky AE, Topp R, Wigglesworth JK, Harsha DM, Edwards JE. Efficacy of a home-based training program for older adults using elastic tubing. Eur J Appl Physiol Occup Physiol 1994 69: 316-320. [PubMed]

30. Hupperets MDW, Verhagen EALM, van Mechelen W. Effect of unsupervised home based proprioceptive training on recurrences of ankle sprain: randomised controlled trial. BMJ 2009 339: b2684. PubMed]

31. Berger L, Martinie P, Livain T, Bergeau J, Rougier P. Immediate effects of physiotherapy session of lower limb by balneotherapy on postural control. Ann Readapt Med Phys 2006 49: 37-43. [PubMed] 